\title{
Measurement of Dielectric Behavior of Fertilized Soil at Microwave Frequency
}

\author{
Vivek Yadav, Anil Kumar, Sudeep Sharan \& AK Sinha \\ Gyrotron Laboratory, Central Electronics Engineering Research Institute (CEERI) \\ Pilani 333031, India \\ E-mail: vivek.ceeri@gmail.com; aksinha@ceeri.ernet.in \\ M Yadav, VK Gupta \& RA Jangid \\ Microwave research laboratory, Raj Rishi Collège, Alwar 301001, India
}

\begin{abstract}
The paper presents the experimental results which have been carried out for understanding the behavior of solid dielectrics in the form of soil at microwave frequency and check the dielectric and electric response of some fertilizers in soil in presence of microwave energy. A simple and rapid measurement method (two point method) was used to determine the dielectric constant at microwave frequency. The microwave beam generated by microwave source was reflected by the dielectric sample which was placed in rectangular waveguide and fundamental mode was considered. The microwave source reflection klystron of frequency range of X- band was used. The soil samples were prepared by mixing different concentrations of some fertilizers in soil. The soils were taken from 'Behror Alwar (RAJ)' and different fertilizers 'Urea, Shree Ram-33, Shree Ram-50P, D.A.P., Mosaic' were taken from market.
\end{abstract}

Keywords: Dielectric constant, Dielectric loss, a.c. conductivity, Relaxation time, Reflection, Two point method

\section{Introduction}

The behavior of dielectric materials in presence of electromagnetic (EM) field is entirely different from that in presence of direct current field. In order to understand the behavior of dielectric materials under the action of electromagnetic fields, one has to investigate its interaction in such EM field. In the presence of an alternative electric field, the dielectric materials get polarized along the field direction. The degree of polarization depends upon the applied EM power and the nature of material itself (1). The static dielectric constant and dipole moment values are the measure of the polarization of the material at low frequencies. However, presence of high frequency field, there exist a time lag in the attainment of equilibrium in system with the changing field and hence an anomalous dispersion (dielectric constant decrease within increase in frequency) takes place. This in turn gives rise to the dielectric relaxation. Spatial and temporal variation of the moisture content in the surface layer of soil is considered important in agriculture. Its knowledge is important for the sowing, development, successful maturation of a crop along with rainfall runoff prediction agricultural yields forecasting and boundary layer heat exchange for meteorological and climates studies(2). The different percentage of fertilizer content in the soil gives rise to a large variation in the dielectric constant. Thus, the knowledge of the variation of dielectric constant of the soil at different fertilizer content is necessary for the efficient use of soil.

\subsection{Role of porosity in soil fertility}

Porosity of the soil greatly helps to judge the moisture movement within the soil. Macro pores allow readily movement of air and water. It does not hold water under normal condition. In contrast, macro pores can hold more water and restrict the movement of air and water in soil. Thus, in sandy soil, inspite of the low total porosity, the movement of air and water is surprisingly rapid because of the dominance of the macro pore spaces. Porosity of soil is easily changed. Any operation that reduces aggregation and decreases the amount of organic matter in the soil, decreases pore space(3). In the present work, dielectric constants of the loamy sand soils with different concentration of different fertilizers content and fixed moisture content were measured at fixed frequency $9.8 \mathrm{GHz}$ in the laboratory condition.

\section{Material and method}

The two-point method of measuring dielectric constant is used for the measurements of dielectric properties. An X-band microwave bench operating at $9.8 \mathrm{GHz}$ in the TE10 mode with slotted section was used and the shift of minima is needed 
in this technique. The soil samples were prepared by mixing different concentrations of some fertilizers in soil. The soils were taken from 'Behror, Alwar (RAJ)' and different fertilizers 'Urea, Shree Ram-33, Shree Ram-50P, D.A.P., Mosaic' were taken from market. First, the soil sample was oven dried and then weighed. Then measured quantity of water (1.5ml) and different quantity (according to agriculture department) of different fertilizers was added and allowed four hours to facilitate internal drainage, subsequent homogenous mixing and settlement. The dielectric constant ( $(\varepsilon)$ and dielectric loss $(\varepsilon ")$ of the samples were measured after determining the weight of the sample at every reading.

From the measurement of dielectric constant and dielectric loss, other electric parameters as a.c. conductivity $(\sigma)$, and relaxation time $(\tau)$ can be obtained (4).

$$
\sigma=\omega \varepsilon_{0} \varepsilon "
$$

And

$$
\tau=\varepsilon " / \omega \varepsilon
$$

Where

$\omega$ is angular frequency, $\mathrm{f}=9.8 \mathrm{GHz}$

$\varepsilon_{0}$ is permittivity of free space.

\section{Results and Discussion}

\subsection{Dielectric constant variation}

The variation in values of dielectric constant and dielectric loss with percentage of fertilizers content are measured and plotted in fig.1,2,3,4 and 5, Similarly, the a.c. electric conductivity and relaxation time with variation of percentage fertilizers content are plotted in fig.6,7,8,9 and10. By measurements it is observed that there is a very little variation in the dielectric constant of soil with fertilizers. Another feature is that the higher fertilizer contents have higher dielectric constant and higher dielectric loss in soil as compared to that low fertilizer content at a given frequency. The value of dielectric constant ( $\left.\varepsilon^{\prime}\right)$ and dielectric loss ( $\varepsilon$ ") increases with fertilizer contents. This slow increase is due to the presence of fertilizers. When the polar molecules are very large, then in presence of high frequency electromagnetic field, the rotary motion of polar molecules is not sufficiently rapid to attain the equilibrium with the field. Therefore, the displacement current acquires conductance dissipation energy. Thus, the a.c. conductivity is increase with dielectric loss. The relaxation time is increase due to increasing hindrance to the process of polarization.

\subsection{Fertility variation}

The fertilizers increase the pore space of the soil (3). Due to more pore space the dielectric constant ( $\left.\varepsilon^{\prime}\right)$ increases and the fertility of soil is also increased. The different types of fertilizers have different organic components. The soil is also affected by these different organic components. Fig.11, shows comparative increasing in dielectric constant $(\varepsilon ')$ of different types of fertilizers. According to this graph, the dielectric constant of soil with SHREE RAM-33 is more than other and it increases speedily as compared to others. The dielectric constant of soil with D.A.P. is less than others and it increases slowly as compare to others. Therefore, the above experimental results clearly show that SHREE RAM -33 speedily increases the pore space of soil as compared to other fertilizers.

\section{Conclusion}

Study of the properties of dry and fertilized sand soil at microwave frequencies in the laboratory is useful in Agriculture. The good results are obtained for the Dielectric Constant, dielectric loss, a.c. conductivity and relaxation time of different samples at X-band frequencies. The a.c. electrical conductivity and relaxation time depend upon the dielectric loss, which represents attenuation and dispersion. The dielectric properties of the solid dielectrics in the form of fertilized soil are useful in understanding the structural behavior of soil. These studies are also useful for increase the fertility of soil. The result of such studies may not be only important for the understanding of the fundamental nature of the response of the particulate soil to the high frequency electromagnetic fields but also of applied nature, useful to increase the pore space of soil.

\section{Acknowledgement}

The authors are thankful to Dr. Chandra Shekhar, Director, CEERI, for granting permission for publication of this paper. The authors are also thankful to their colleagues of CEERI for helpful interaction.

\section{References}

Aikawa, M. and Ogawa, H. (1980). A new MIC Magic T using coupled Slot lines, IEEE Trans. MTT-28 (6).

Benchimol G, de Bruyne F. A. (1965). Measurements of dielectric constant of solid materials. Electronic measuring and Microwave Notes (pp 5-8), Philips, 2. 
Bottcher, C.J.F. (1952). Theory of electric Polarization, Elsevier Pub. Comp. Ams. Houston, London, 415.

Chaudgari, H.C. and Shinde, V.J. (2008). Dielectric study of moisture laden soils at X-band microwave frequency. IJPS, Vol. 3(3),

Clavin, A. (1955). High Power Ferrite Load Isoltors Trans. Inst. Radio Enggrs. MTT-3.

Dakin, T.W. and works, C.N. (1947). Microwave Dielectric measurements. Journal of Applied Physics, Vol. 18(a), Sept.

Frohilch, H. (1949). Theory of dielectrics. Oxford University Press, London.

Ghose, R.N. (1963). Microwave circuit theory and Analysis.McGraw-Hill Book Co.

Ginzton, E.L. (1957). Microwave Measurements. McGraw-Hill Co. L Inc.

R. Trouch Fedrick and Thomson Louis M. (1993). Soil and Soil fertility. Oxford University Press.

Richard OB. (1963). Laboratory Manual for Microwave Measurements, Vol I, Edn III.

Sucher, M. and Fox, J. (1983). Handbook of Microwave measurements, Vol III, John Wiley \& Sons, Inc., New Yark.

Wang J R and Schmugge T J. (1980). IEEE Trans Geosci Remote Sens (USA), 18, 288.

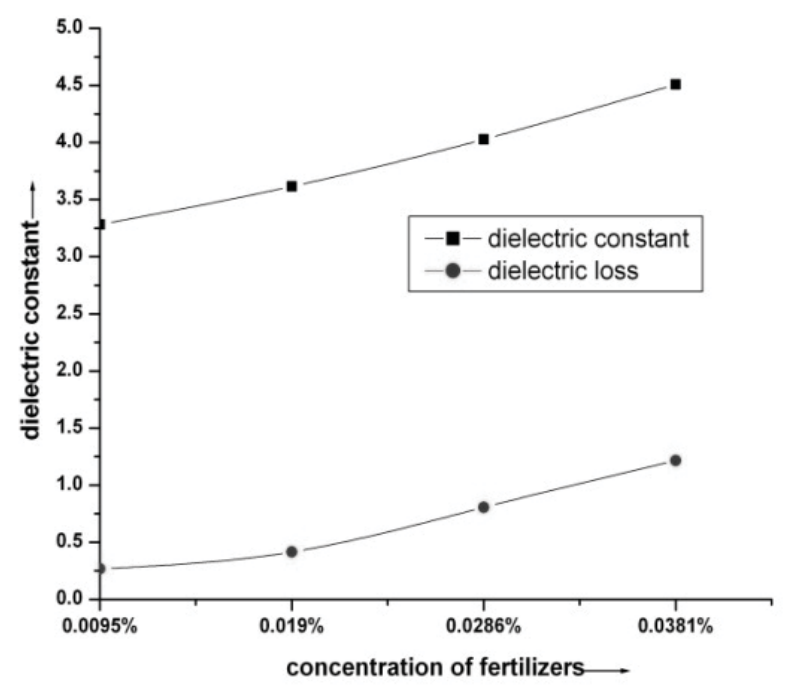

Figure 1. Variation of dielectric constant and dielectric loss with fertilizer content for SHREE RAM-33

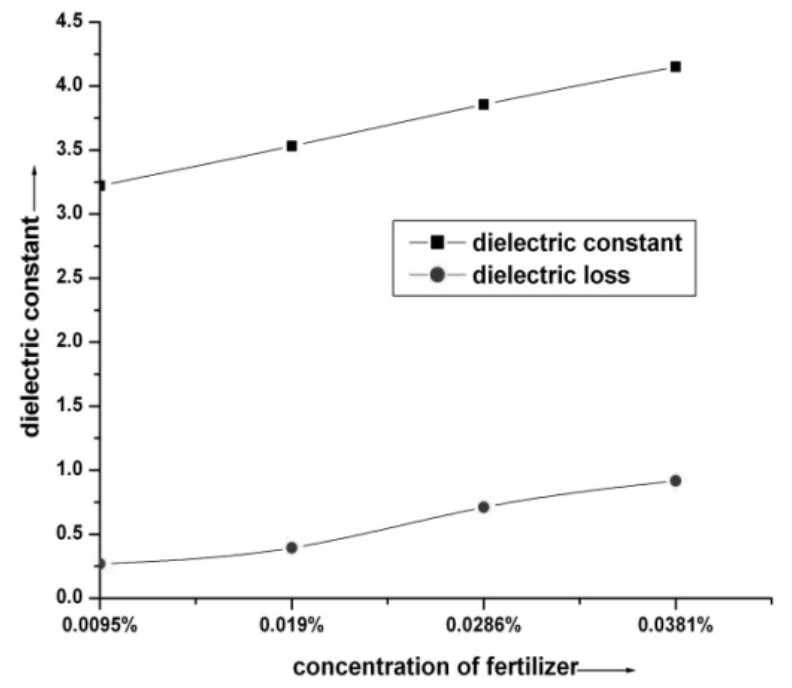

Figure 2. Variation of dielectric constant and dielectric loss with fertilizer content for SHREE RAM-50P 


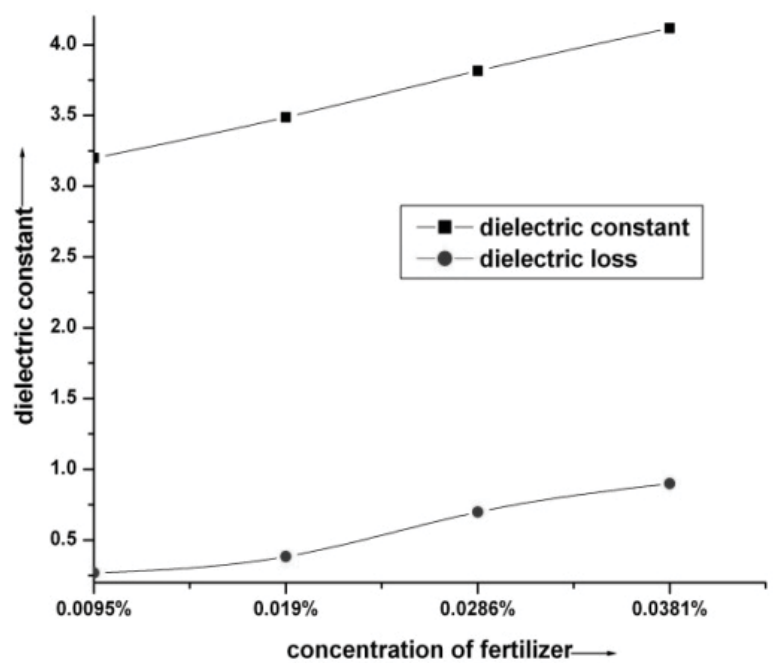

Figure 3. Variation of dielectric constant and dielectric loss with fertilizer content for MOSAIC

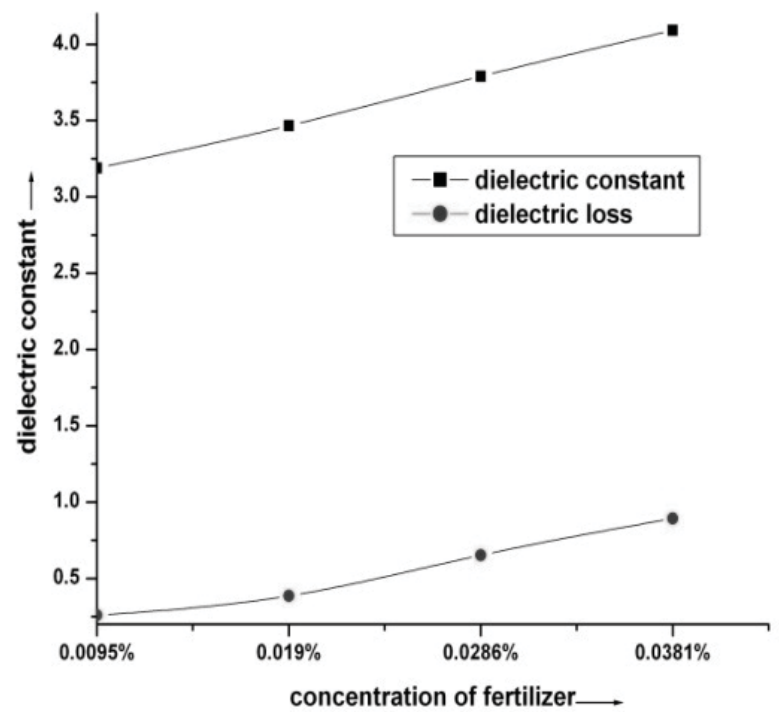

Figure 4. Variation of dielectric constant and dielectric loss with fertilizer content for UREA 


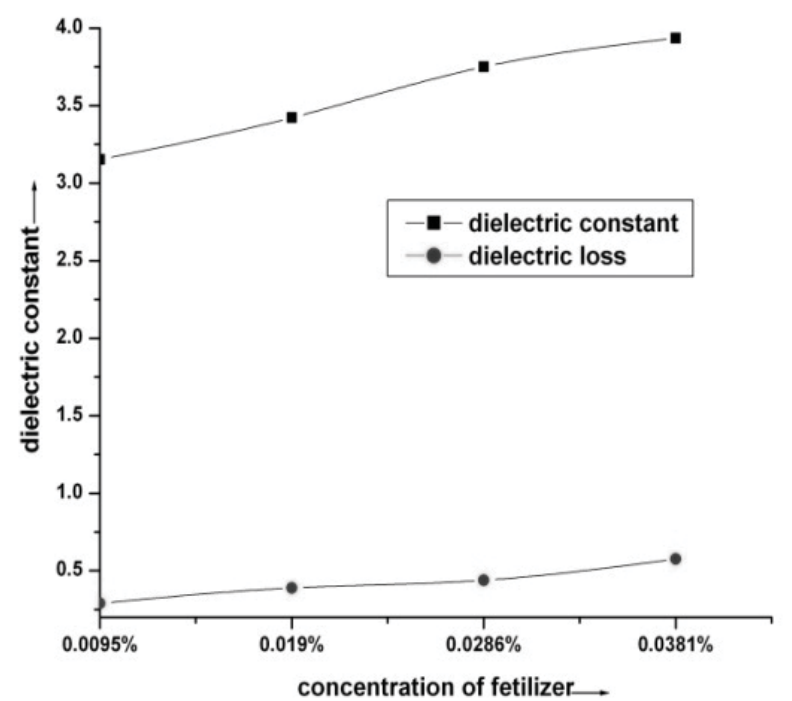

Figure 5. Variation of dielectric constant and dielectric loss with fertilizer content for D.A.P

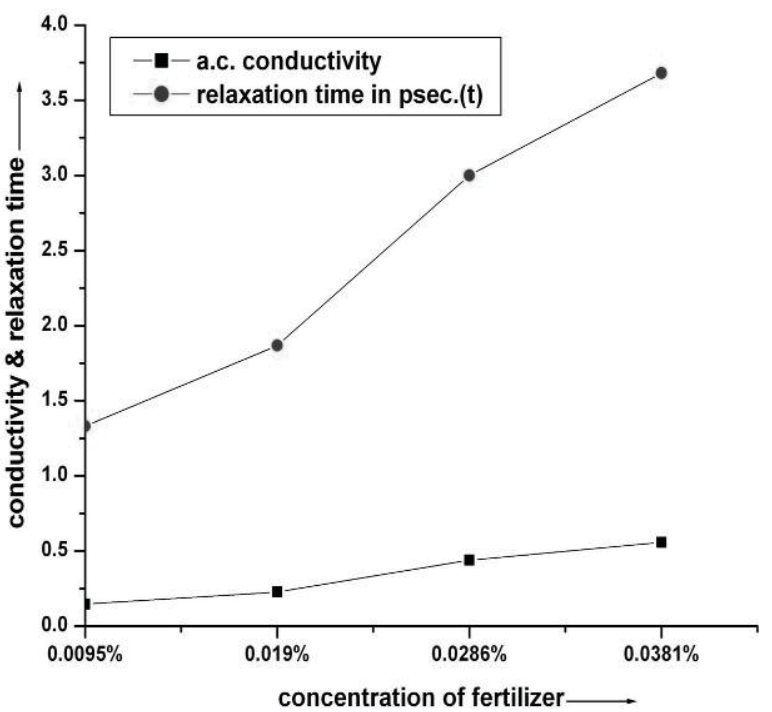

Figure 6. Variation of a.c. conductivity \& relaxation time with fertilizer content for SHREE RAM-33 


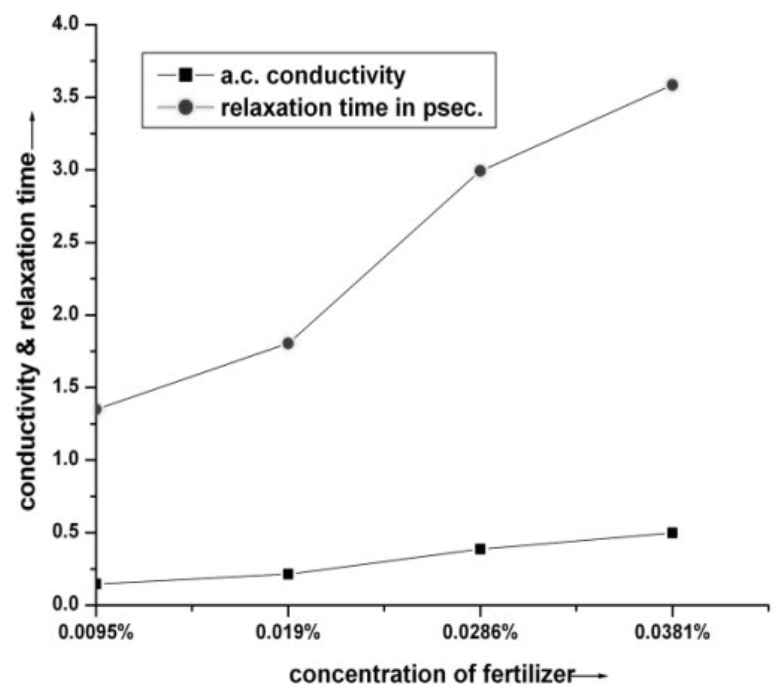

Figure 7. Variation of a.c. conductivity \& relaxation time with fertilizer content for SHREE RAM-50P

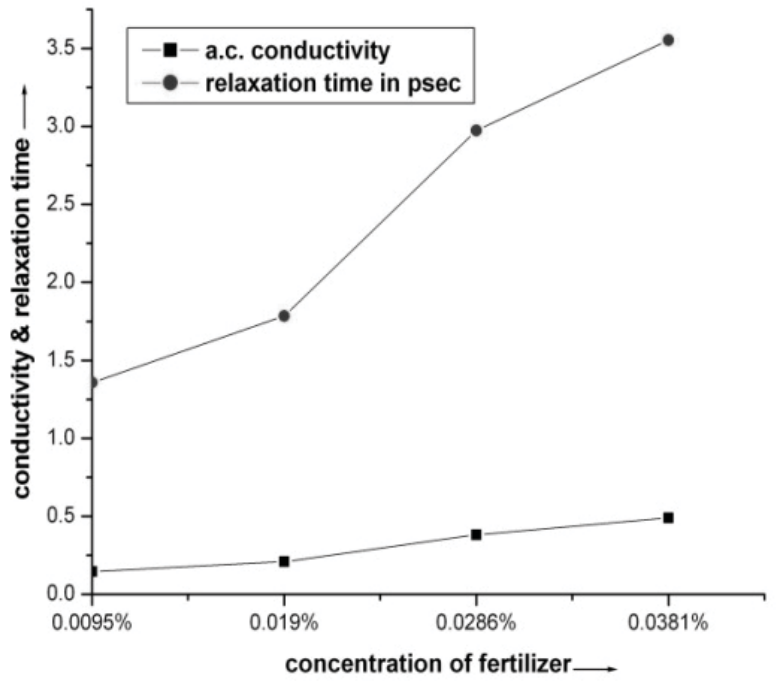

Figure 8. Variation of a.c. conductivity \& relaxation time with fertilizer content for MOSAIC 


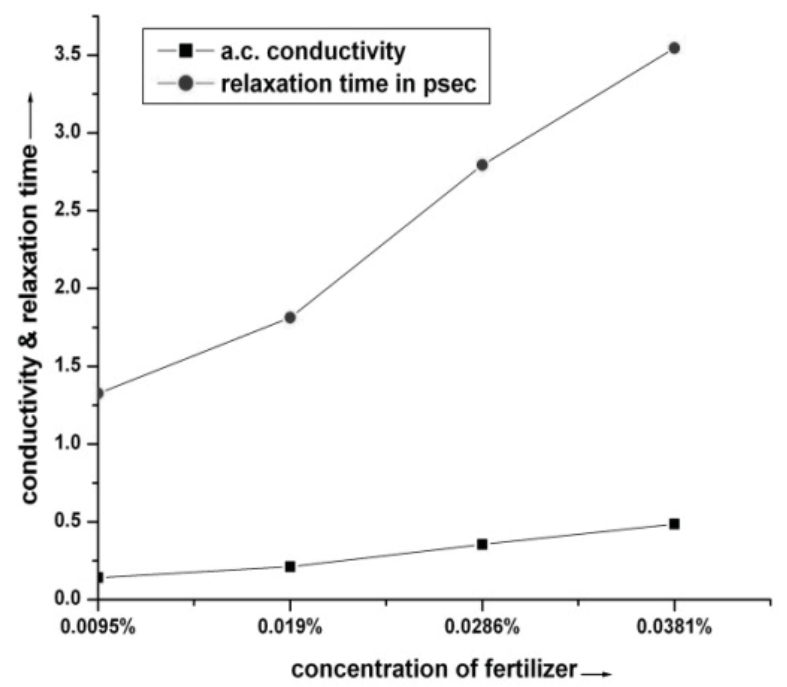

Figure 9. Variation of a.c. conductivity \& relaxation time with fertilizer content for UREA

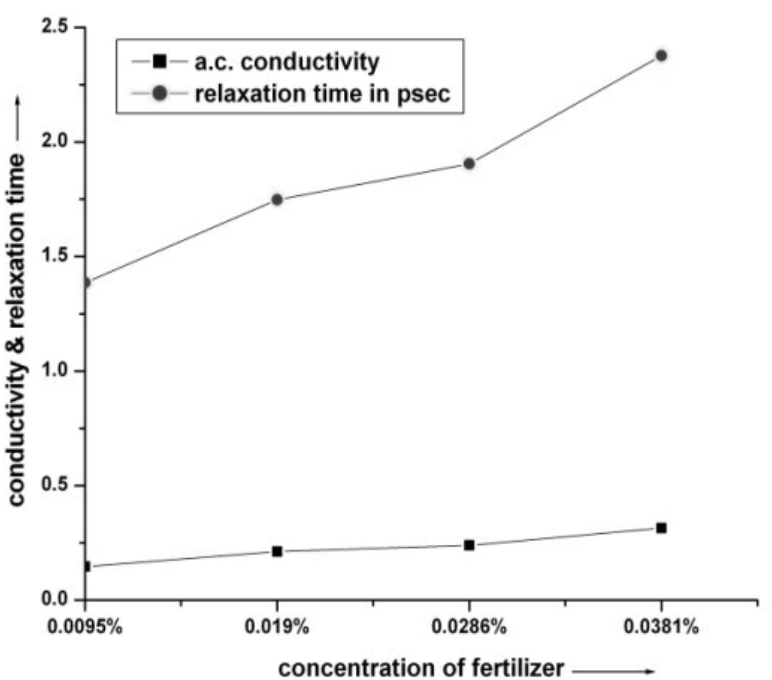

Figure 10. Variation of a.c. conductivity \& relaxation time with fertilizer content for D.A.P 


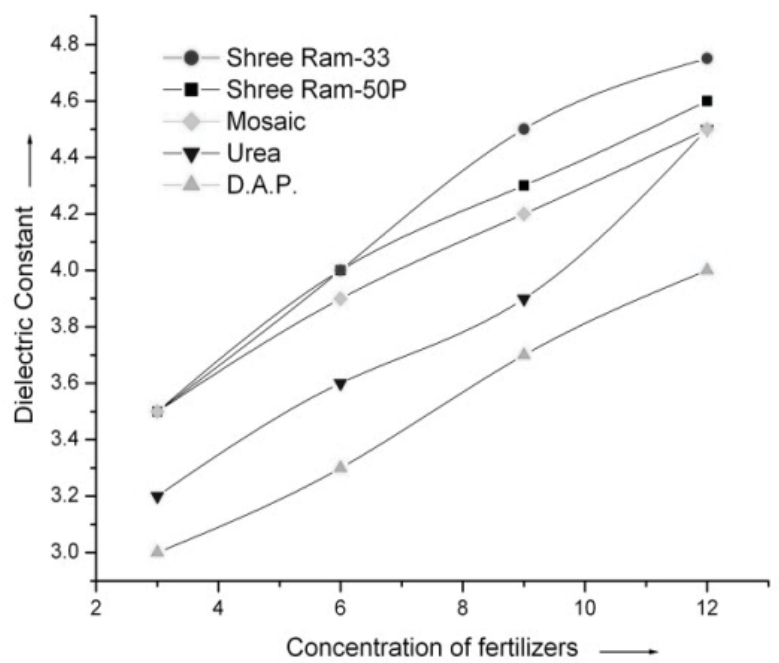

Figure 11. Graph between dielectric constant and concentration of fertilizers 